\title{
Lentiviral vector-mediated co-overexpression of VEGF and Bcl-2 improves mesenchymal stem cell survival and enhances paracrine effects in vitro
}

\author{
XIAOBIN NI ${ }^{1,2}$, CAIWEN OU $^{1}$, JINGBIN GUO $^{1}$, BEI LIU ${ }^{1}$, JIANWU ZHANG ${ }^{1}$, \\ ZHIYE WU ${ }^{1}$, HEKAI LI $^{1}$ and MINSHENG CHEN ${ }^{1}$ \\ ${ }^{1}$ Department of Cardiology, Zhujiang Hospital of Southern Medical University, Guangzhou, Guangdong 510280; \\ ${ }^{2}$ Shantou Central Hospital, Shantou, Guangdong 515031, P.R. China \\ Received February 1, 2016; Accepted May 30, 2017
}

DOI: 10.3892/ijmm.2017.3019

\begin{abstract}
Mesenchymal stem cell (MSC) transplantation has emerged as a promising therapy for ischemic heart disease; however, the low survival rate of transplanted cells limits their therapeutic efficacy. The aim of this study was to investigate whether the dual genetic modification of vascular endothelial growth factor (VEGF) and B-cell lymphoma-2 (Bcl-2) confers a higher expression level of the target genes, better survival and a stronger paracrine effect in MSCs in an adverse environment than the modification of the individual genes. For this purpse, a lentiviral vector was constructed by using a self-cleaving T2A peptide sequence to link and achieve the co-overexpression of VEGF and Bcl-2. Rat MSCs were transfected to obtain cell lines that exhibited a stable overexpression. An in vitro model of oxygen glucose deprivation (OGD) was applied to mimic the ischemic microenvironment, and cell apoptosis, autophagy and the paracrine effects were then determined. Compared with the MSCs in which individual genes were modified and the control MSCs, the MSCs which were subjected to dual genetic modification had a higher expression level of the target genes, a more rapid proliferation, reduced apoptosis, decreased autophagy and an enhanced paracrine effect. Furthermore, the suppression of autophagy was found to contribute to the inhibition of apoptosis in this in vitro OGD model. On the whole, these data indicate that the co-overexpression of VEGF and $\mathrm{Bcl}-2$ protects MSCs in an ischemic environment by inhibiting apoptosis, suppressing autophagy and enhancing the paracrine effects.
\end{abstract}

Correspondence to: Professor Minsheng Chen, Department of Cardiology, Zhujiang Hospital of Southern Medical University, 1023 Shatai Road, Guangzhou, Guangdong 510280, P.R. China E-mail: gzchenminsheng@163.com

Key words: vascular endothelial growth factor, B-cell lymphoma-2, mesenchymal stem cell, apoptosis, autophagy, paracrine effect

\section{Introduction}

Stem cell transplantation has emerged as a promising, alternative treatment for ischemic heart disease. Of the available stem cells, mesenchymal stem cells (MSCs) hae been widely studied and are considered as a leading candidate with many advantages, such as the capacity for self-renewal, a multilineage differentiation potential, low immunogenicity, immunosuppressive properties and low tumorigenicity $(1,2)$. Transdifferentiation and paracrine signaling are considered to be the principal mechanisms of the effects of MSCs in the treatment of ischemic heart disease (3).

It is evident that the first, vital step of cell therapy is to enable the stem cells to reach the targeted ischemic location and to successfully promote survival, which could be partly responsible for the modest benefits of stem cell therapy observed in a clinical trial setting (4). Undoubtedly, the improvement of the engraftment and survival of the transplanted stem cells is vital. Many efforts have been made in that regard, mainly focusing on the improvement of the microenvironment and the enhancement of cellular resistance to a hostile environment via pre-treatment with growth factors or cytokines, hypoxic preconditioning, and genetic modification with anti-apoptotic, angiogenetic and other genes (5). An improved therapeutic efficacy has been observed in animals with transplanted genetically modified MSCs compared to animals that have been transplanted with untreated MSCs. This process is known as cell-based gene therapy (6). Recent data have demonstrated that the modification of the angiogenetic gene vascular endothelial growth factor (VEGF) in MSCs exerts a better angiogenetic and paracrine effect (7), while the modification of the anti-apoptotic gene, B-cell lymphoma-2 (Bcl-2), confers improved survival (8). Importantly, compared to the untreated MSCs, VEGF- and Bcl-2-modified cells have been shown to improve cardiac function. Moreover, an interaction which could promote the mutual expression of VEGF and Bcl-2 has been observed in several studies (8-10).

Given the information described above, we hypothesized that the combined overexpression of VEGF and Bcl-2 could confer much greater resistance to the MSCs in an adverse environment. 


\section{Materials and methods}

Recombinant lentiviral vector construction. In order to confer stable overexpression, a lentiviral vector was used for gene delivery $(11,12)$. The plasmid, pLV-CMV-EF1-fLuc-T2A-puro, purchased from HanBio Technology Co., Ltd. (Shanghai, China) was used to construct the recombinant lentiviral vectors. The human VEGF gene (NM_001171626.1) and human Bcl-2 gene (NM_000633) were polymerase chain reaction (PCR)-amplified from a complementary deoxyribonucleic acid (cDNA) library. A self-cleaving T2A peptide sequence was employed to link and achieve the co-overexpression of VEGF and Bcl-2 (13). A Bcl-2-T2A-VEGF fragment was obtained by overlapping PCR. PCR primers were designed and the $E c o \mathrm{R} 1$ and $X h o 1$ restriction endonuclease sites were introduced into the plasmid as follows: VEGF, 5'-ACACGAATTCATGAACTTTCTGCTGTCTTGGGT-3' (forward) and 5'-ATTGCTCGAGTCACCGCCTCGGCTT GTCACATC-3' (reverse); Bcl-2, 5'-GAATTCATGGCGCACG CTGGGAGAACAGG-3' (forward) and 5'-ATTGCTCGAG TCACTTGTGGCCCAGATAGGCACC-3' (reverse); Bcl-2T2A-VEGF, 5'-GAATTCATGGCGCACGCTGGGAGAAC AGG-3' (forward); Fusion-R1, 5'-CACGTCACCGCATGTTA GAAGACTTCCTCTGCCCTCCTTGTGGCCCAGATAG-3' and Fusion-F2, 5'-ACATGCGGTGACGTGGAGGAGAATC CCGGCCCTATGAACTTTCTGCTGTCTTG-3' and 5'-TTG CTCGAGTTCACCGCCTCGGCTTGTCACATC-3' (forward). Following the addition of $10 \mu \mathrm{l}$ of the PCR products onto a $1 \%$ agarose gel with ethidium bromide $(0.5 \mathrm{mg} / \mathrm{ml})$ and electrophoresis, images were captured by ultraviolet transillumination. The plasmid was doubly digested with EcoR1 and Xho1 (NEB, Ipswich, MA, USA). The PCR products and plasmid were purified, and they were then ligated and the ligation mixture was transformed into competent Escherichia coli DH5a cells (Invitrogen, Carlsbad, CA, USA). Clones were selected for PCR validation, and the recombinant plasmid was extracted for sequencing. The lentivirus packaging system consists of 3 plasmids: pLV-CMV-EF1-fLuc-T2A-puro, pSPAX2 and pMD2G (HanBio Technology Co., Ltd.). These 3 plasmids were co-transfected into the 293T cell line (HanBio Technology Co., Ltd.) according to the instructions of the manufacturer of Lipofectamine 2000 (Invitrogen) after the endotoxin was removed. The cells were transferred to a complete medium after $8 \mathrm{~h}$ of co-transfection. The supernatant was harvested and concentrated after $48 \mathrm{~h}$ of incubation. The viral titer was determined and calibrated in the 293T cell line. The 293T cell culture medium was then centrifuged and concentrated to obtain a lentivirus solution, which was kept in a collection cup at $-80^{\circ} \mathrm{C}$. The 3 lentiviral vectors were named Lv-VEGF, Lv-Bcl-2 and Lv-BV. The control lentiviral vector encoding green fluorescent protein (GFP) was purchased from HanBio Technology Co., Ltd.

MSC preparation, cell transfection and the generation of cell lines with stable expression. OriCell ${ }^{\mathrm{TM}}$ Sprague-Dawley (SD) rat MSCs, which had been primarily isolated, cultured and passaged no more than twice, were purchased from Cyagen Biosciences Inc. (Santa Clara, CA, USA). The MSCs were cultured according to the manufacturer's instructions. Briefly, the MSCs were seeded in cell culture flasks (Corning Inc.,
Corning, NY, USA) in complete medium containing low glucose-Dulbecco's modified Eagle's medium (DMEM), $10 \%$ fetal bovine serum (both from Gibco, Grand Island, $\mathrm{NY}, \mathrm{USA}), 100 \mathrm{U} / \mathrm{ml}$ penicillin and $100 \mu \mathrm{g} / \mathrm{ml}$ streptomycin sulphate (Gibco) at $37^{\circ} \mathrm{C}$ in a humidified atmosphere in a $5 \% \mathrm{CO}_{2}$ and $95 \%$ air incubator (Thermo Fisher Scientific, San Jose, CA, USA). When $80 \%$ confluence was reached, the cells were detached using $0.25 \%$ trypsin-EDTA (Gibco) and subcultured at a 1:2 ratio. The 4th passage MSCs were seeded into 6-well plates (Coster, Cambridge, MA, USA) at $1 \times 10^{4}$ cells $/ \mathrm{cm}^{2}$. Lv-GFP was used as a control. When the cells were $50 \%$ confluent, they were infected with Lv-GFP at a multiplicity of infection (MOI) of 10, 20, 50,100 or 200. GFP expression was observed under a fluorescence microscope (Leica, Wetzlar, Germany) after $48 \mathrm{~h}$. An MOI=100 was selected for subsequent experiments, which was adequate for sufficient genetic overexpression in the infected cells with minimum damage. The 4 lentiviruses Lv-GFP, Lv-VEGF, Lv-Bcl-2 and Lv-BV were each added separately into the culture medium at an MOI of 100 . Transfections were performed for $24 \mathrm{~h}$ at $37^{\circ} \mathrm{C}$, and the virus-containing medium was then replaced with fresh complete medium and the cells were incubated for a further $48 \mathrm{~h}$. Stably transfected cells were selected by the addition of $2 \mu \mathrm{g} / \mathrm{ml}$ puromycin (Sigma-Aldrich, St. Louis, MO, USA) to the medium, which was changed every 2 days. Selection was terminated when the uninfected control cells were completely dead. The 4 stably expressing cell lines were designated as MSC-GFP, MSC-VEGF, MSC-Bcl-2 and MSC-BV.

Cell proliferation assay. The proliferation of stably expressing cell lines under normal culture conditions was assessed using a Cell Counting kit-8 (CCK-8; Dojindo Laboratories, Kumamoto, Japan) according to the manufacturer's instructions. For culturing, the cells were plated in 96-well plates (Coster) at $5 \times 10^{3}$ cells/well. CCK-8 solution $(10 \mu \mathrm{l})$ was added to each well followed by incubation at $37^{\circ} \mathrm{C}$ for $1 \mathrm{~h}$. The optical density (OD) at $450 \mathrm{~nm}$ was measured using a Synergy 2 multi-mode microplate reader (BioTek, Winooski, VT, USA) at 7 days.

Reverse transcription-quantitative (real-time) $P C R(R T-q P C R)$. RT-qPCR was performed to analyze the messenger ribonucleic acid (mRNA) expression levels of VEGF and Bcl-2. TRIzol reagent (Invitrogen) was used to extract the RNA from the MSCs and then reverse transcribed into cDNA using the GoScript reverse transcription system (Promega, Madison, WI, USA) following the manufacturer's instructions. FastStart Universal SYBR-Green Master (Rox) (Roche Applied Science, Mannheim, Germany) was used for quantitative (real-time) PCR analysis. The primer sequences used in this experiment were: VEGF forward, 5'-GCACTGGACCCTGGCTTTACT-3 and reverse, 5'-ATGGGACTTCTGCTCTCCTTCTG-3'; Bcl-2 forward, 5'-GGGAGATCGTGATGAAGTACATACA-3' and reverse, 5'-GCACAGCGGGCATTGGGTTG-3'; glyceraldehyde 3-phosphate dehydrogenase (GAPDH) forward, 5'-TCCACTCACGGCAAATTCAAC-3' and reverse, 5'-GTAG ACTCCACGACATACTCAGC-3'. The PCR-amplified mRNA was quantified and the results were normalized against GAPDH expression. The $2^{-\Delta \Delta C q}$ method was used to calculate the relative mRNA expression level. 
Western blot analysis. A western blot assay was performed. Briefly, the cell lysate supernatants were collected. A Bradford assay was used to measure the protein concentration. Protein samples $(20-25 \mu \mathrm{g})$ were separated by sodium dodecyl sulfate polyacrylamide gel electrophoresis (Boster, Wuhan, China) and transferred onto polyvinylidene difluoride membranes (Millipore, Milford, MA USA). The membranes were probed overnight with primary antibodies as follows: mouse anti-VEGF (1:200 dilution, cat. no. Ab1316; Abcam, Cambridge, UK), rabbit anti-Bcl-2 (1:1,000 dilution, cat. no. 2870; Cell Signaling Technology, Danvers, MA, USA), rabbit anti-GAPDH (1:1,000 dilution, cat. no. 2118; Cell Signaling Technology), mouse anti-Bax (1:1,000 dilution, cat. no. sc-7480; Santa Cruz Biotechnology, Santa Cruz, CA, USA), rabbit anti-cleaved-caspase-3 (1:1,000 dilution, cat. no. 9661), rabbit anti-LC3 (1:1,000 dilution, cat. no. 2775), rabbit anti-Beclin-1 (1:1,000, cat. no. 3495) and rabbit ant-p62 (1:1,000 dilution, cat. no. 8025, all from Cell Signaling Technology). Diluted secondary antibodies [HRP goat anti-mouse IgG (1:40,000 dilution, cat. no. 115-035-003) and HRP goat antirabbit IgG (1:10,000 dilution, cat. no. 111-035-003; both from Jackson ImmunoResearch Laboratory Inc., West Groove, PA, USA)] were used to detect the corresponding primary antibodies. Further analysis was carried out using ImageJ software (NIH, Bethesda, MD, USA) to quantify the protein bands.

In vitro model of oxygen glucose deprivation (OGD). To mimic the ischemic microenvironment that the transplanted cells encounter in vivo, the MSCs were exposed to OGD conditions at $37^{\circ} \mathrm{C}, 95 \% \mathrm{~N}_{2}, 5 \% \mathrm{CO}_{2}$ in glucose-free DMEM medium (Gibco). Four MSC lines of the same passage were seeded into individual 6 -well plates at a density of $5 \times 10^{4} / \mathrm{cm}^{2}$. Following $24 \mathrm{~h}$ of culture, the cells were washed 3 times with phosphate-buffered saline (PBS) gently and were then cultured under OGD conditions for $12 \mathrm{~h}$. A plate of MSC-GFP was simultaneously cultured under normal conditions as a negative control (MSC-NC).

Flow cytometric analysis of apoptosis. A cell apoptosis assay was performed using an Annexin V-FITC/propidium iodide (PI) apoptosis detection kit (Invitrogen) according to the manufacturer's instructions. Briefly, the cells were collected and washed twice with PBS and then suspended in $300 \mu$ l of binding buffer. Annexin V solution ( $5 \mu \mathrm{l})$ was added to the cell suspension and incubated for $15 \mathrm{~min}$ in the dark at room temperature. Subsequently, $200 \mu 1$ of blinding buffer and $5 \mu 1$ of PI were added and the cell suspension was immediately analyzed on a flow cytometer (Accuri ${ }^{\mathrm{TM}}$ C6; BD Biosciences, San Jose, CA, USA). Annexin $\mathrm{V}^{-} / \mathrm{PI}^{-}$were viable cells, Annexin $\mathrm{V}^{+} / \mathrm{PI}^{-}$cells were early apoptotic cells, and Annexin $\mathrm{V}^{+} / \mathrm{PI}^{+}$cells were late apoptotic or dead cells.

Suppression of autophagy in the MSC-BV group under OGD conditions. The cells in the MSC-BV group were exposed to $12 \mathrm{~h}$ of OGD conditions in the absence or presence of $5 \mathrm{mmol} / \mathrm{l}$ of 3-methyladenine (3-MA; Sigma-Aldrich), an autophagy inhibitor. Flow cytometric analysis and western blot analysis for LC3, Beclin-1, p62, cleaved-caspase-3 and GAPDH was carried out to assess the effects of the suppression of autophagy on the apoptosis of the cells in the MSC-BV group under OGD conditions.

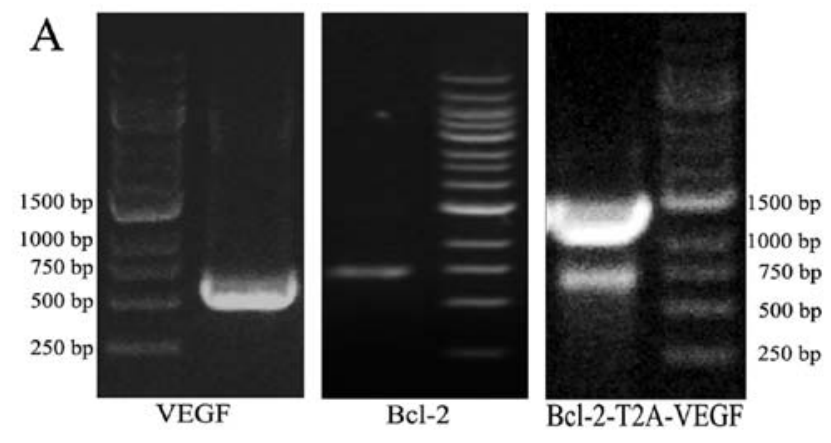

B

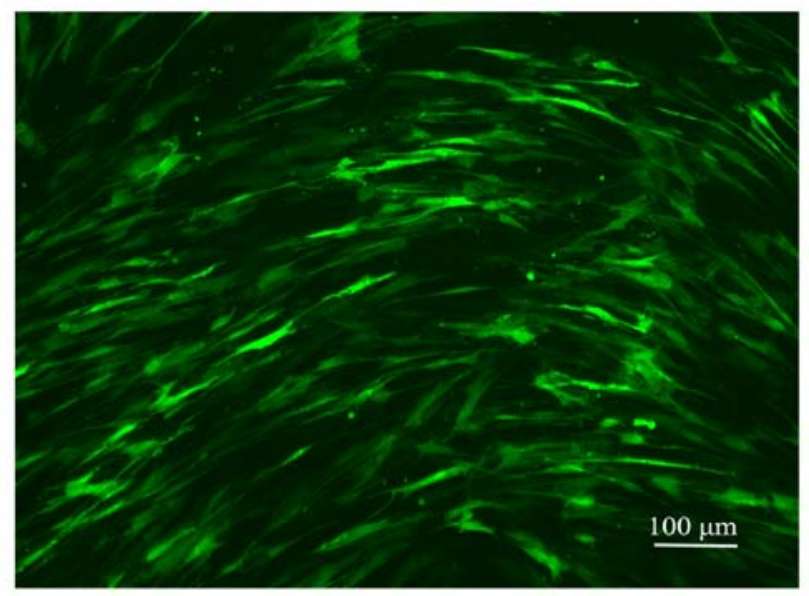

Figure 1. PCR of the target gene fragments and the infection rate of Lv-green fluorescent protein (GFP) in mesenchymal stem cells (MSCs). (A) Agarose gel electrophoresis revealed that the PCR amplified target gene fragments were in the expected positions, 596 bp of vascular endothelial growth factor (VEGF), 736 bp of Bcl-2 and 1363 bp of Bcl-2-T2A-VEGF. (B) The infection rate of Lv-GFP in the MSCs at an MOI of 100 was observed under a fluorescence microscope at $48 \mathrm{~h}$ following infection.

Enzyme-linked immunosorbent assay (ELISA). To determine whether the genetic modification of MCSs affects paracrine signaling following culturing under OGD conditions, an ELISA was performed to detect the concentration of VEGF, basic fibroblast growth factor (bFGF), hepatocyte growth factor (HGF) and insulin-like growth factor-1 (IGF-1) in the culture supernatant using the appropriate ELISA kits (RapidBio, Winnetka, CA, USA) according to the manufacturer's instructions. Supernatant samples were collected from the 4 treatment groups. The OD value at $450 \mathrm{~nm}$ was detected using an ELISA reader (BioTek).

Statistical analysis. The data are expressed as the means \pm standard deviation (SD). Statistical analysis was performed using SPSS for Windows version 20.0 (SPSS, Inc., Chicago, IL, USA). Comparisons between more than 2 groups were performed by a one-way analysis of variance (ANOVA), followed by a post hoc Tukey test. Comparisons between 2 groups were performed using the Student's t-text. A value of $\mathrm{P}<0.05$ was considered to indicate a statistically significant difference.

\section{Results}

Recombinant lentiviral vector construction. Following PCR amplification, agarose gel electrophoresis revealed that the target gene fragments were in the expected positions (Fig. 1A). 
A
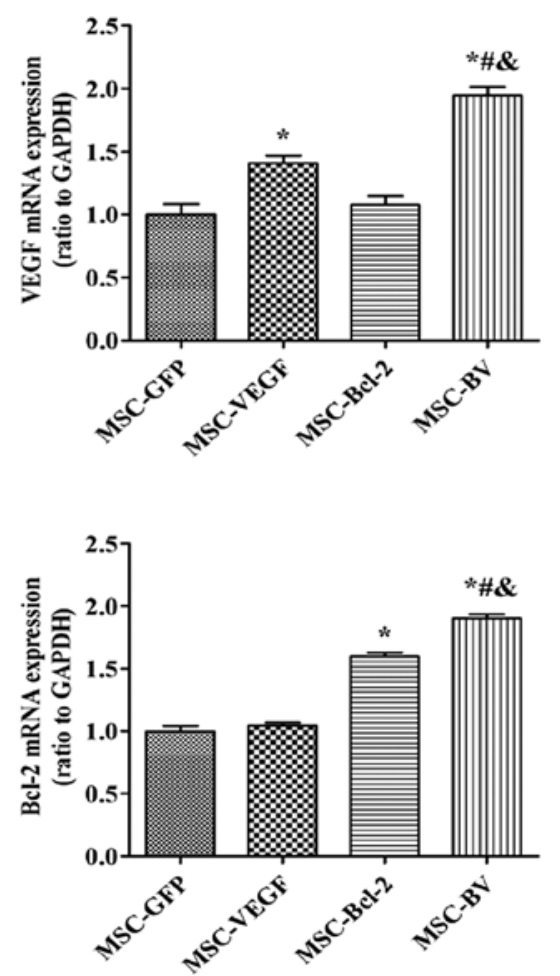

B
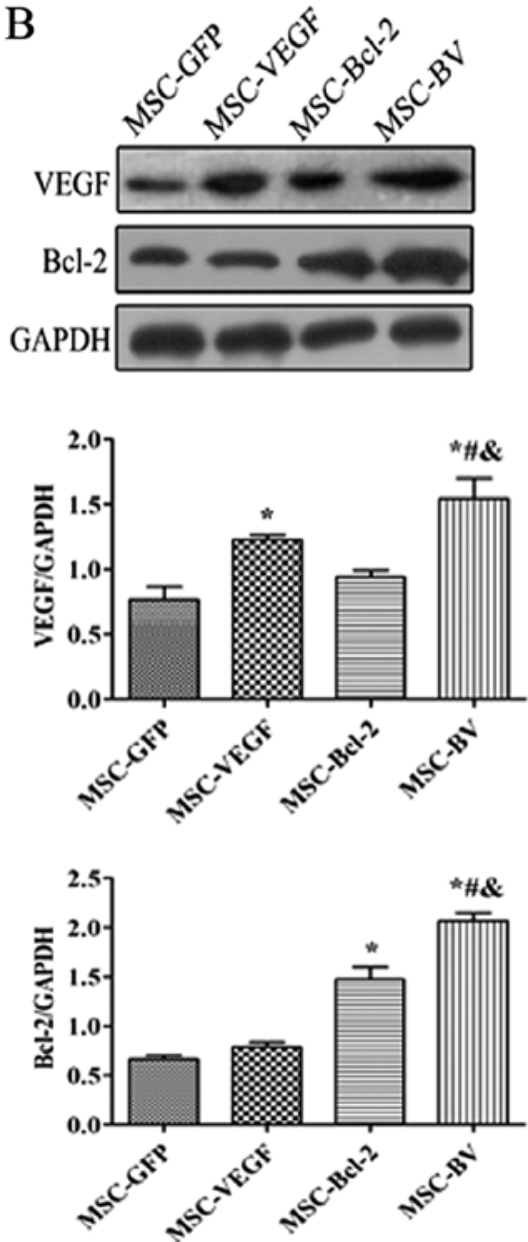

Figure 2. Target gene expression. (A) RT-qPCR detected the mRNA expression levels of the target genes. (B) A representative western blot and quantitative analysis of the vascular endothelial growth factor (VEGF), Bcl-2 and glyceraldehyde 3-phosphate dehydrogenase (GAPDH) levels. ${ }^{*} \mathrm{P}<0.05$ compared to mesenchymal stem cell (MSC)-green fluorescent protein (GFP) ; ${ }^{*} \mathrm{P}<0.05$ compared to MSC-VEGF group; ${ }^{\circledR} \mathrm{P}<0.05$ compared to MSC-Bcl-2 group. Each of the experiments was repeated 5 times, $\mathrm{n}=5$.

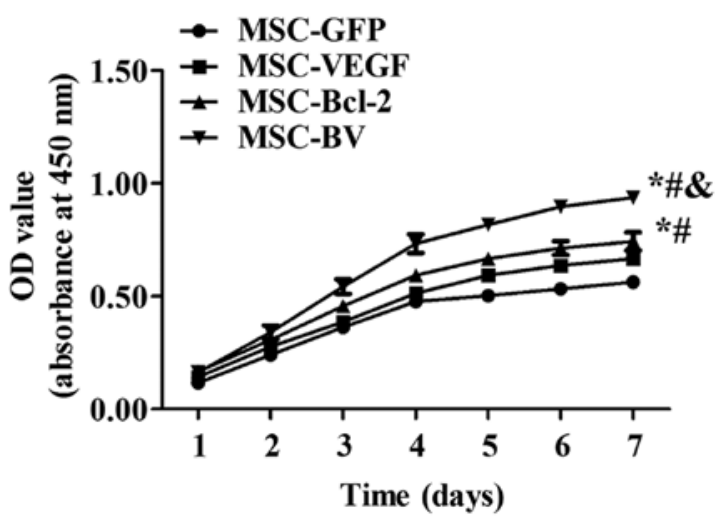

Figure 3. Cell proliferation. The growth curve graph of the 4 cell lines with stable expression over a period of 7 days was examined using the cell counting kit-8 (CCK-8). ${ }^{*} \mathrm{P}<0.05$ compared to mesenchymal stem cell (MSC)-green fluorescent protein (GFP) group; ${ }^{\#} \mathrm{P}<0.05$ compared to MSC-vascular endothelial growth factor (VEGF) group; ${ }^{\&} \mathrm{P}<0.05$ compared to $\mathrm{MSC}-\mathrm{Bcl}-2$ group. Each of the experiments was repeated 5 times, $\mathrm{n}=5$.

Sequencing confirmed that the gene fragments were correctly subcloned into the vector (data not shown). The lentiviral vectors were successfully constructed, and the viral titer was $1 \times 10^{8} \mathrm{TU} / \mathrm{ml}$ after packaging.

The 4 cell lines with stable gene expression, target gene expression and cell proliferation. The infection rate of Lv-GFP in the MSCs at an MOI of 100 was approximately $70 \%$ (Fig. 1B). Following 3 rounds of puromycin addition, stable cell lines that expressed the target genes were selected. RT-qPCR indicated that the mRNA expression levels of both VEGF and Bcl-2 in the MSC-BV group were markedly higher than those in the other cell groups (Fig. 2A). Western blot analysis further confirmed the highest protein expression of both VEGF and Bcl-2 in the MSC-BV group (Fig. 2B). A CCK-8 assay revealed that the cells in the MSC-BV group proliferated more rapidly than the 3 other cell lines over a period of 7 days under normal culture conditions (Fig. 3).

Co-overexpression of VEGF and Bcl-2 inhibits the OGD-induced apoptosis of MSCs. To examine the anti-apoptotic activity of MSCs co-overexpressing VEGF and Bcl-2 under an adverse environment, the cells in the MSC-GFP, MSC-VEGF, MSC-Bcl-2 and MSC-BV groups were individually cultured under OGD conditions for $12 \mathrm{~h}$. An Annexin V-FITC/PI 

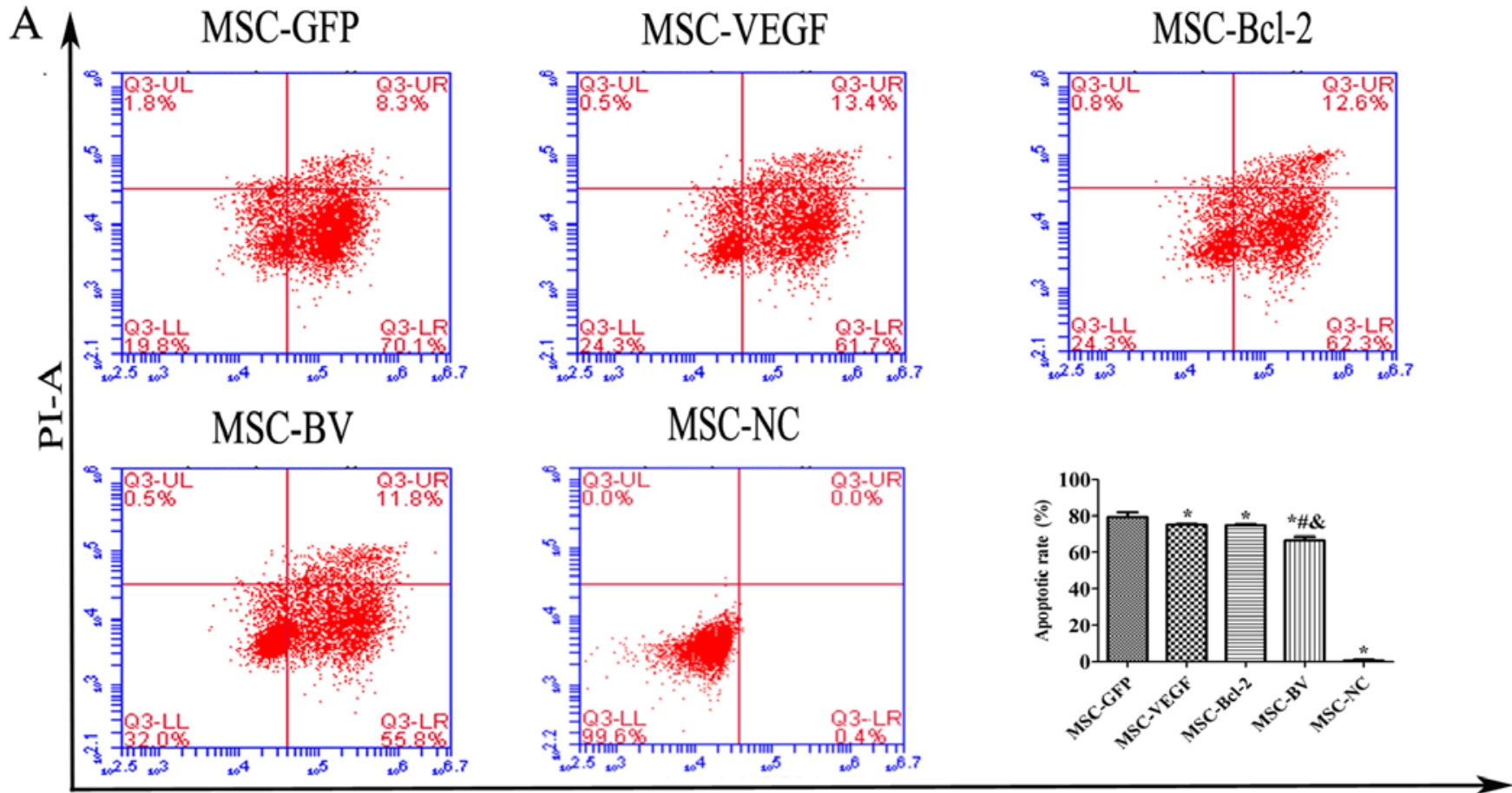

\section{Annexin V FITC-A}
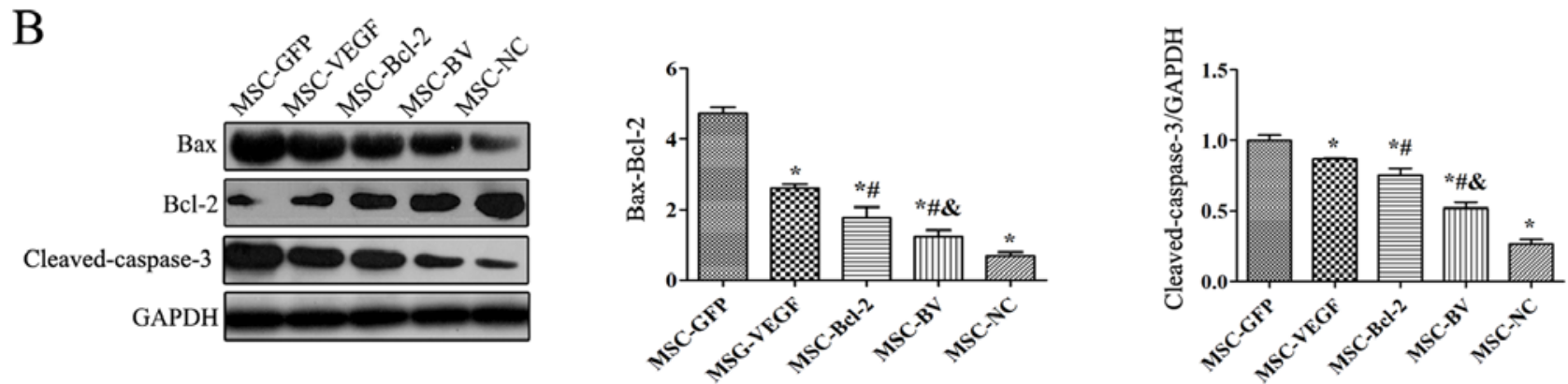

Figure 4. The influence of the dual genetic modification on the apoptosis of mesenchymal stem cells (MSCs) under oxygen glucose deprivation (OGD) conditions. (A) The apoptotic rate in the MSCs was determined by flow cytometry using FITC-Annexin V/PI double staining. Upper left quadrant, necrotic cells; lower left quadrant, live cells; upper right quadrant, late apoptotic cells; lower right quadrant, early apoptotic cells. (B) Representative western blot and quantitative analysis of the Bax, Bcl-2, cleaved-caspase-3 and glyceraldehyde 3-phosphate dehydrogenase (GAPDH) levels to indicate apoptosis. "P $<0.05$ compared to MSC-green fluorescent protein (GFP) group; ${ }^{\#} \mathrm{P}<0.05$ compared to MSC-vascular endothelial growth factor (VEGF) group; ${ }^{\&} \mathrm{P}<0.05$ compared to MSC-Bcl-2 group. Each of the experiments was repeated 5 times, $n=5$.

flow cytometric assay revealed that the apoptotic rate of the cells in the MSC-BV group was significantly lower than that of the 3 other cell groups (Fig. 4A). Western blot results also revealed that the lowest $\mathrm{Bax} / \mathrm{Bcl}-2$ ratio and cleaved-caspase- 3 protein expression were observed in the cells in the MSC-BV group (Fig. 4B).

Co-overexpression of VEGF and Bcl-2 suppresses the $O G D$-induced autophagy of MSCs. The results of western blot analysis revealed that following exposure to OGD, the levels of LC3II/I and Beclin-1 in the MSC-GFP group were markedly increased compared to the MSC-NC group. However, their expression levels in the MSC-VEGF and MSC-Bcl-2 groups were relatively lower than those in the MSC-GFP group, while the expression levels were lowest in the MSC-BV group. The expression of p62 in the MSC-GFP group was markedly decreased compared to the level in the MSC-NC group. However, p62 expression in the MSC-VEGF and MSC-Bcl-2 groups was relatively higher than that in the MSC-GFP group, while the expression was the highest in the MSC-BV group (Fig. 5).

Suppression of autophagy contributes to the inhibition of the apoptosis of MSCs under OGD conditions. The Annexin V-FITC/PI flow cytometric assay revealed that following exposure to OGD, the cells in the MSC-BV + 3-MA 

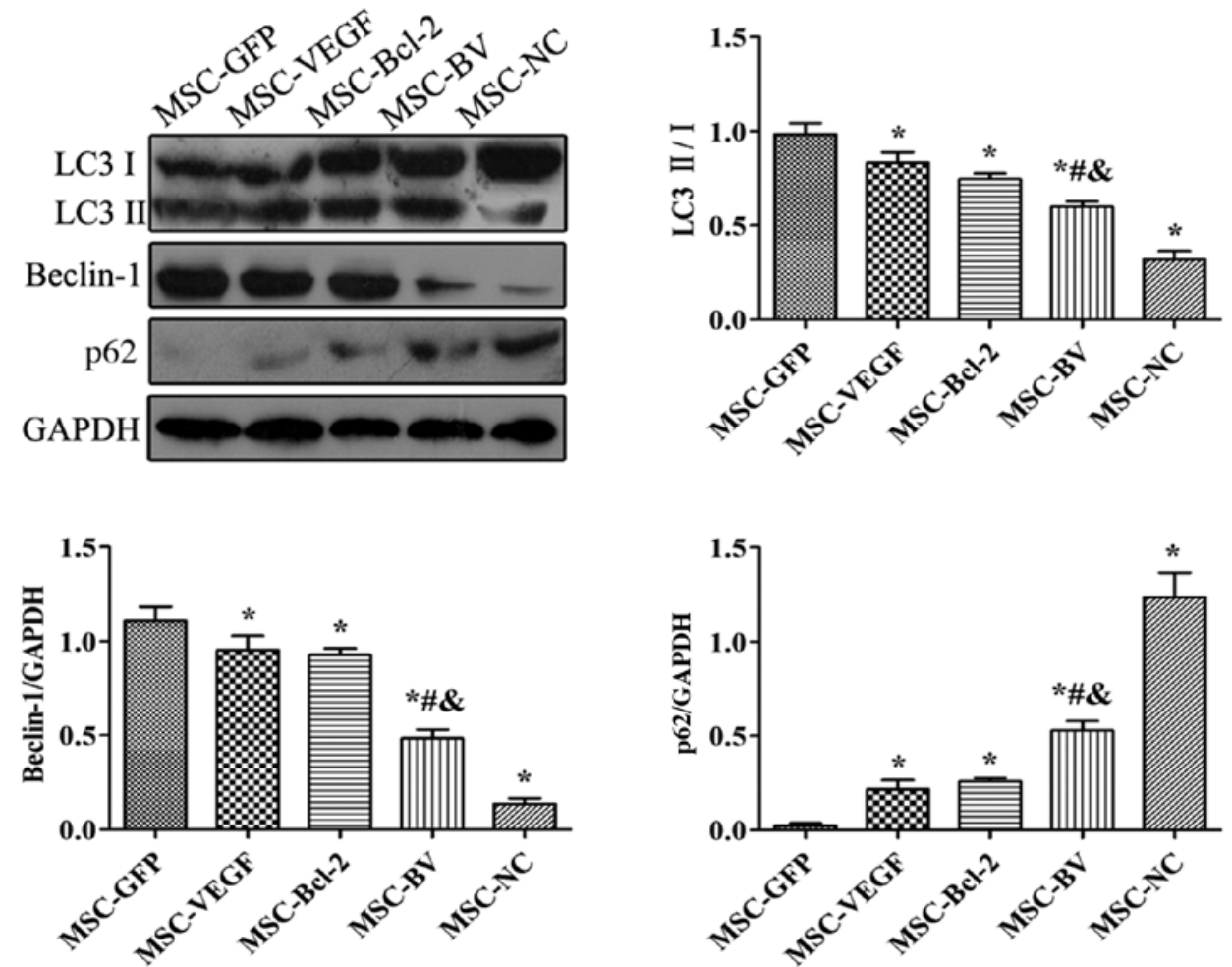

Figure 5. The influence of the dual genetic modification on autophagy. A representative western blot and quantitative analysis of the LC3, Beclin-1, p62 and glyceraldehyde 3-phosphate dehydrogenase (GAPDH) levels to indicate autophagy. ${ }^{*} \mathrm{P}<0.05$ compared to mesenchymal stem cell (MSC)-green fluorescent protein (GFP) group; ${ }^{*} \mathrm{P}<0.05$ compared to MSC-vascular endothelial growth factor (VEGF) group; ${ }^{\circledR} \mathrm{P}<0.05$ compared to MSC-Bcl-2 group. Each of the experiments was repeated 5 times, $\mathrm{n}=5$.

A

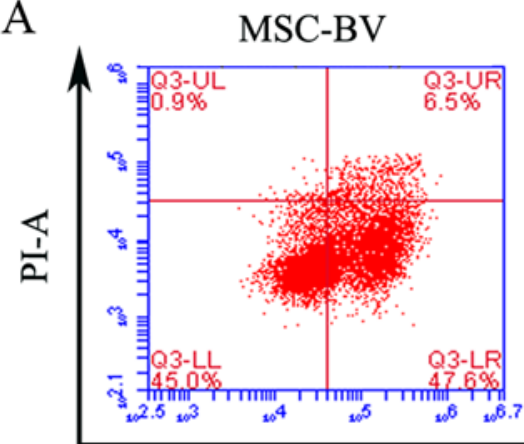

B

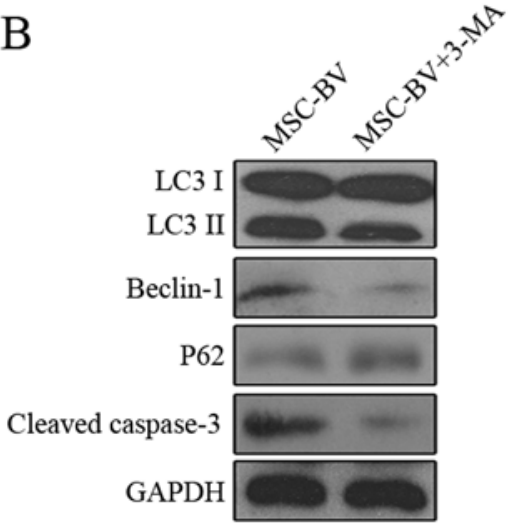

MSC-BV+3-MA

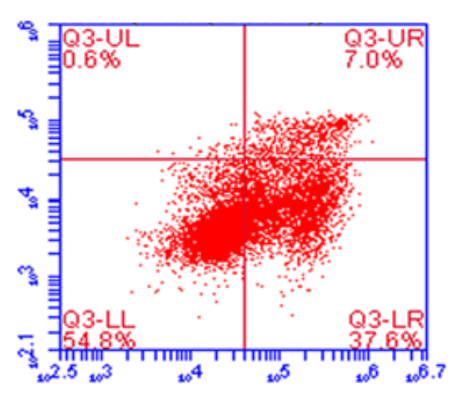

Annexin V FITC-A

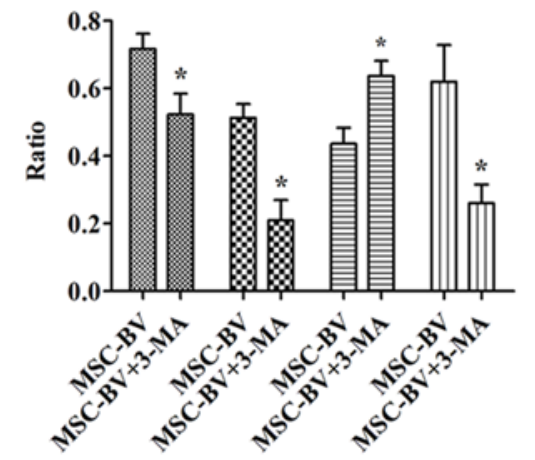

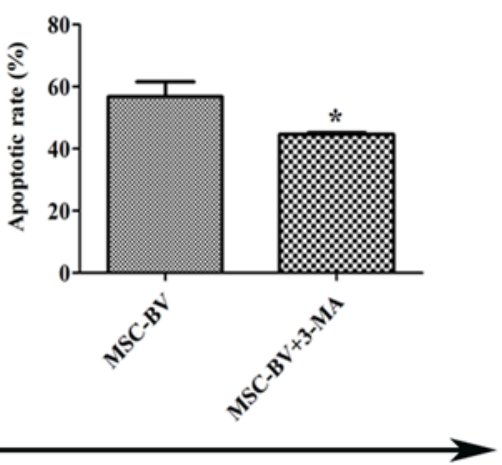

LC3 II/I

$\otimes$ Beclin-1/GAPDH

P62/GAPDH

血 Cleaved caspase-3/GAPDH

Figure 6. Suppression of autophagy contributes to the inhibition of the apoptosis of mesenchymal stem cells (MSCs) under oxygen glucose deprivation (OGD) conditions. (A) Flow cytometry using FITC-Annexin V/PI double staining detected the apoptotic rate of cells in the MSC-BV group in the absence or presence of 3-MA under OGD conditions. Upper left quadrant, necrotic cells; lower left quadrant, live cells; upper right quadrant, late apoptotic cells; lower right quadrant, early apoptotic cells. (B) A representative western blot and quantitative analysis of LC3, beclin-1, p62 and cleaved-caspase-3 to indicate autophagy and apoptosis in cells in the MSC-BV group treated with 3-MA added for the suppression of autophagy under OGD conditions. * $<<0.05$ compared to MSC-BV group. Each of the experiments was repeated 5 times, $\mathrm{n}=5$. 

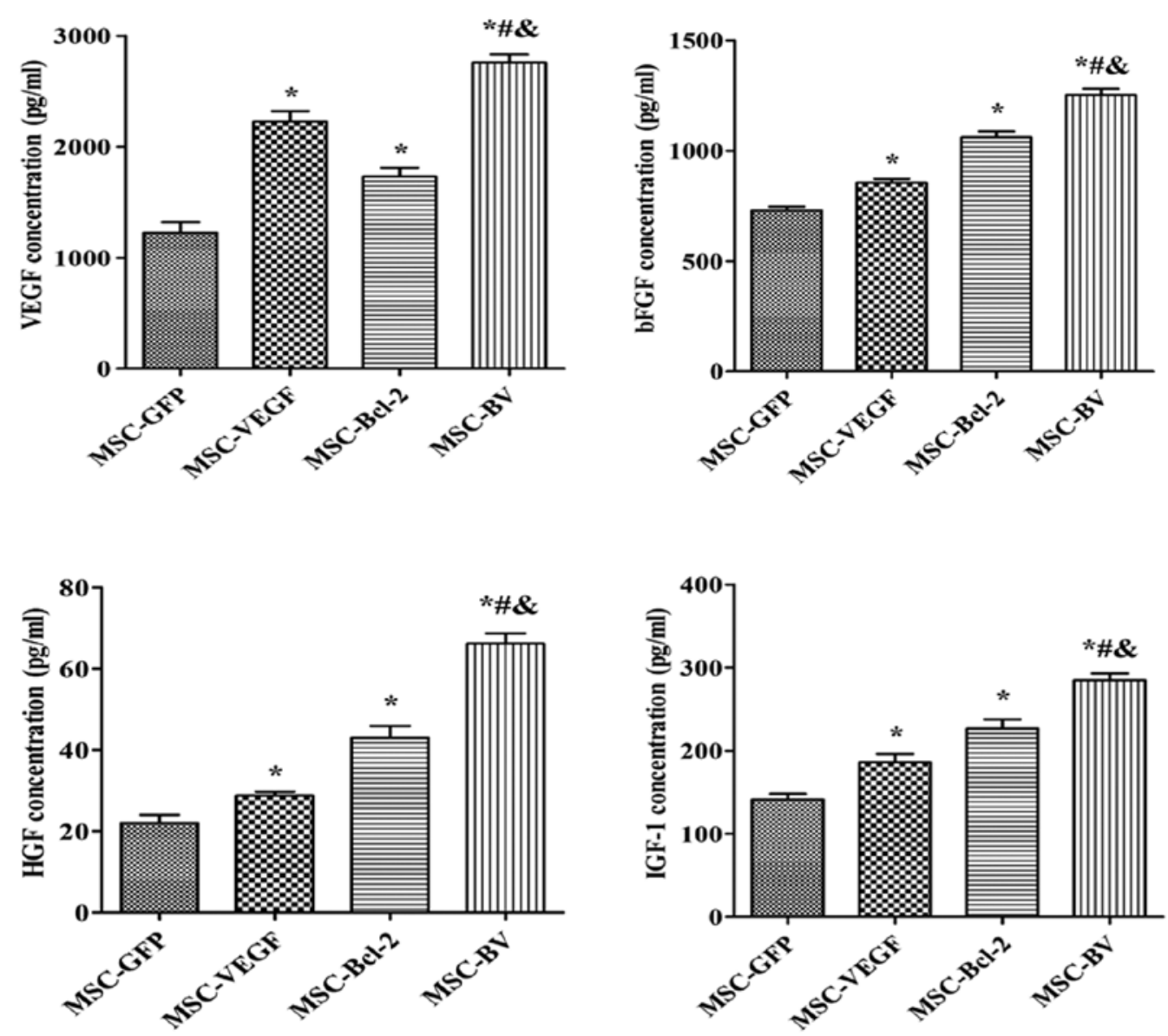

Figure 7. Paracrine signaling in mesenchymal stem cells (MSCs) under oxygen glucose deprivation (OGD) conditions. The concentration of vascular endothelial growth factor (VEGF), basic fibroblast growth factor (bFGF), hepatocyte growth factor (HGF) and insulin-like growth factor 1 (IGF-1) of culture supernatant were detected by enzyme-linked immuno sorbent assay (ELISA). " $\mathrm{P}<0.05$ compared to MSC-green fluorescent protein (GFP) group; ${ }^{*} \mathrm{P}<0.05$ compared to MSC-VEGF group; ${ }^{\circledR} \mathrm{P}<0.05$ compared to MSC-Bcl-2 group. Each of the experiments was repeated 5 times, $\mathrm{n}=5$.

group had a lower apoptotic rate than the cells in the MSC-BV group (Fig. 6A). Western blot analysis revealed that the levels of LC3II/I, Beclin-1 and cleaved-caspase-3 in the MSC-BV + 3-MA group were markedly decreased compared to those in the MSC-BV group, while the expression of p62 was increased (Fig. 6B).

Co-overexpression of VEGF and Bcl-2 enhances the paracrine effects of MSCs under OGD conditions. An ELISA was used to detect the concentration of VEGF, bFGF, HGF and IGF-1. Consistent trends in the levels of these growth factors were observed. The cells in the MSC-BV group had the highest level of growth factors out of all the groups (Fig. 7).

\section{Discussion}

In this study, we first constructed a lentiviral vector using a self-cleaving T2A peptide sequence to link and achieve the co-overexpression of the VEGF and Bcl-2 genes, and we then transfected the MSCs and generated cell lines that stably overexpressed VEGF and Bcl-2. We subsequently confirmed that the VEGF and Bcl-2 dual genetically modified MSCs had higher mRNA and protein expression levels and a more rapid proliferation than the MSCs in which single genes had been modified. Moreover, our study also demonstrated that the dual genetic modification of VEGF and Bcl-2 in the MSCs led to a more pronounced and additive self-protection effect than the modification of single genes in MSCs in an in vitro ischemic model (OGD), which was indicated by the inhibition of apoptosis, the suppression of autophagy and the enhancement of the paracrine effects. We even found that the suppression of autophagy may have contributed to the inhibition of apoptosis in MSCs under OGD conditions. To the best of our knowledge, this is the first study that combines the simultaneous modification of the angiogenic VEGF gene and the anti-apoptotic Bcl-2 gene in MSCs. We confirmed that this combined strategy endowed the MSCs with a better capacity to confront an adverse environment than the modification of single genes, and an even stronger paracrine effect.

Stem cell transplantation has been widely studied and is considered a promising therapy for ischemic heart disease. Although our results were encouraging, many preclinical and clinical studies have shown that the poor survival of transplanted cells can limit their efficacy. Therefore, measures aimed at enhancing the tolerance of MSCs to a hostile environment are vital for cell therapy, and gene modification may represent a potential strategy. It has been demonstrated that the overexpression of anti-apoptotic genes, such as Bcl-2, Akt, Hsp-27, Hsp-20 and survivin is relatively effective in improving the survival of MSCs in an adverse environment, improving cardiac func- 
tion (8,14-17). VEGF has been used as a potent therapeutic reagent in the treatment of ischemia via the induction of angiogenesis in MSCs. VEGF-modified MSCs have been shown to possess an enhanced repair capacity due to the induction of angiogenesis and an enhanced paracrine effect (7,18-21). Of note, the expression of VEGF in MSCs has been shown to be upregulated by Bcl-2 modification under hypoxic conditions (8). In addition, a similar regulation of $\mathrm{Bcl}-2$ expression by VEGF in hypoxic cancer cells has been observed (9). The involvement of the MAPK signaling pathways may be partly responsible for the interaction VEGF and Bcl-2 (10). Based on this information, it is reasonable to hypothesize that combining the VEGF and Bcl-2 genes in MSCs may enhance survival, and improve angiogenesis and enhance the paracrine effects. In this study, our results confirmed this hypothesis. Under normal culture conditions, dual genetically modified MSCs had a higher overexpression level of VEGF and Bcl-2. Under OGD conditions, dual genetically modified MSCs exhibited higher survival rate and stronger paracrine effect.

A previous study even confirmed that the strategy of combining an anti-apoptotic and an angiogenetic gene in MSCs was feasible (22). In that study, the authors achieved the co-overexpression of Akt and Ang-1 using two adenoviral vectors, which confirmed that the co-overexpression led to better cytoprotection in the in vitro OGD model, and showed that dually genetically modified MSCs transplanted into animals with myocardial infarction could significantly improve cardiac function by improving cellular survival and angiogenesis. To a certain extent, our results from our in vitro experiments are in accordance with the findings of that study. However, compared to the previous study, our study has several advantages. First, the interaction of VEGF and Bcl-2 was taken into account in our selection of genes. It is not simply an additive effect, but a mutual promotion. Second, our study used a lentiviral vector to deliver the genes as this vector has a better transfection efficiency and is less toxic to cells than the adenoviral vector (23). Importantly, the use of lentivirus enables final cell lines to stably co-overexpress target genes, which allowed us to better observe the effects of the genetic modification than with the transient expression of the adenoviral vector. Moreover, a self-cleaving T2A peptide sequence was used to link the two genes and place them in a single lentiviral vector, which reduced the viral dosage and ensured the equivalent expression level.

The Bcl-2 gene is also known to play an important role in cellular autophagy (24); thus, in this study, we also carried out simultaneous western blot analyses to investigate several autophagy-related proteins in a preliminary assessment of the effects of the dual genetic modification under OGD conditions on autophagy in MSCs. Our results suggested that the activation of autophagy in MSCs following $12 \mathrm{~h}$ of exposure to OGD may be suppressed by dual genetic modification. We then further confirmed that the suppression of autophagy contributed to the inhibition of apoptosis in the OGD-exposed cells in the MSC-BV group.

Therefore, the inhibition of apoptosis in the dually modified cells may be partly achieved by the indirect suppression of autophagy. The association between autophagy and apoptosis is controversial. Initially, autophagy was found to favor cell survival (25), but later it was also found to play a role in cell death. Excessive and prolonged autophagy may promote cell death $(26,27)$. In this study, both the autophagy and apoptosis of MSCs induced by OGD may contribute to cell death, and was suppressed by the dual genetic modification.

The paracrine effect is an important mechanism in the use of MSCs in the treatment of ischemic heart disease and has been widely used as a target for improvement via various means, including but not limited to, cellular factors. It has been shown that VEGF-, bFGF-, HGF- and IGF-1-modified MSCs can significantly enhance the paracrine effects (28-30). Our results from the ELISA detection of four classical growth factors confirmed that the MSCs dually modified with VEGF and Bcl-2 exhibited an enhanced paracrine effect that improved MSC resistance to a harsh microenvironment, further playing a protective role against ischemic disease.

Despite these encouraging results, some challenges remain to be addressed further. First, the benefits from dually modified VEGF and Bcl-2 MSCs must be further confirmed in an animal study. Second, the signaling pathways involved in the inhibition of apoptosis must be further clarified. Third, the association and signaling pathways between VEGF and Bcl-2 in MSCs should be further elucidated. Fourth, the role of autophagy in MSCs in a hostile environment and its association with apoptosis must be further investigated.

In conclusion, in this study, we constructed a lentiviral vector that overexpressed the VEGF and Bcl-2 genes simultaneously, and confirmed that the dual genetic modification of the MSCs had a higher expression than singly modified MSCs. Such a dual genetic modification strategy successfully made the MSCs much more resistant to a hostile environment via the inhibition of apoptosis, the suppression of autophagy and the enhancement of paracrine signaling.

\section{Acknowledgements}

This study was supported by the National Natural Science Foundation of China (grant no. 31271053).

\section{References}

1. Wang X, Zhang J, Zhang F, Li J, Li Y, Tan Z, Hu J, Qi Y, Li Q and Yan B: The clinical status of stem cell therapy for ischemic cardiomyopathy. Stem Cells Int 2015: 135023, 2015.

2. Wang XJ and Li QP: The roles of mesenchymal stem cells (MSCs) therapy in ischemic heart diseases. Biochem Biophys Res Commun 359: 189-193, 2007.

3. Duran JM, Makarewich CA, Sharp TE, Starosta T, Zhu F, Hoffman NE, Chiba Y, Madesh M, Berretta RM, Kubo H, et al: Bone-derived stem cells repair the heart after myocardial infarction through transdifferentiation and paracrine signaling mechanisms. Circ Res 113: 539-552, 2013.

4. Young PP and Schäfer R: Cell-based therapies for cardiac disease: A cellular therapist's perspective. Transfusion 55: 441-451, quiz 440, 2015

5. Lee S, Choi E, Cha MJ and Hwang KC: Cell adhesion and long-term survival of transplanted mesenchymal stem cells: A prerequisite for cell therapy. Oxid Med Cell Longev 2015: 632902, 2015.

6. Penn MS and Mangi AA: Genetic enhancement of stem cell engraftment, survival, and efficacy. Circ Res 102: 1471-1482, 2008.

7. Matsumoto R, Omura T, Yoshiyama M, Hayashi T, Inamoto S, Koh KR, Ohta K, Izumi Y, Nakamura Y, Akioka K, et al: Vascular endothelial growth factor-expressing mesenchymal stem cell transplantation for the treatment of acute myocardial infarction. Arterioscler Thromb Vasc Biol 25: 1168-1173, 2005. 
8. Li W, Ma N, Ong LL, Nesselmann C, Klopsch C, Ladilov Y, Furlani D, Piechaczek C, Moebius JM, Lützow K, et al: Bcl-2 engineered MSCs inhibited apoptosis and improved heart function. Stem Cells 25: 2118-2127, 2007.

9. Baek JH, Jang JE, Kang CM, Chung HY, Kim ND and Kim KW: Hypoxia-induced VEGF enhances tumor survivability via suppression of serum deprivation-induced apoptosis Oncogene 19: 4621-4631, 2000.

10. Wang D, Weng Q, Zhang L, He Q and Yang B: VEGF and Bcl-2 interact via MAPKs signaling pathway in the response to hypoxia in neuroblastoma. Cell Mol Neurobiol 29: 391-401, 2009.

11. Shearer RF and Saunders DN: Experimental design for stable genetic manipulation in mammalian cell lines: Lentivirus and alternatives. Genes Cells 20: 1-10, 2015.

12. Sakuma T, Barry MA and Ikeda Y: Lentiviral vectors: Basic to translational. Biochem J 443: 603-618, 2012.

13. Ibrahimi A, Vande Velde G, Reumers V, Toelen J, Thiry I, Vandeputte C, Vets S, Deroose C, Bormans G, Baekelandt V, et al: Highly efficient multicistronic lentiviral vectors with peptide 2A sequences. Hum Gene Ther 20: 845-860, 2009.

14. McGinley LM, McMahon J, Stocca A, Duffy A, Flynn A, O'Toole D and O'Brien T: Mesenchymal stem cell survival in the infarcted heart is enhanced by lentivirus vector-mediated heat shock protein 27 expression. Hum Gene Ther 24: 840-851, 2013

15. Wang X, Zhao T, Huang W, Wang T, Qian J, Xu M, Kranias EG, Wang Y and Fan GC: Hsp20-engineered mesenchymal stem cells are resistant to oxidative stress via enhanced activation of Akt and increased secretion of growth factors. Stem Cells 27: 3021-3031, 2009.

16. Mangi AA, Noiseux N, Kong D, He H, Rezvani M, Ingwall JS and Dzau VJ: Mesenchymal stem cells modified with Akt prevent remodeling and restore performance of infarcted hearts. Nat Med 9: 1195-1201, 2003.

17. Fan L, Lin C, Zhuo S, Chen L, Liu N, Luo Y, Fang J, Huang Z, Lin $\mathrm{Y}$ and Chen J: Transplantation with survivin-engineered mesenchymal stem cells results in better prognosis in a rat model of myocardial infarction. Eur J Heart Fail 11: 1023-1030, 2009

18. Tang YL, Zhao Q, Qin X, Shen L, Cheng L, Ge J and Phillips MI: Paracrine action enhances the effects of autologous mesenchymal stem cell transplantation on vascular regeneration in rat model of myocardial infarction. Ann Thorac Surg 80: 229-237, 2005.

19. Banai S, Jaklitsch MT, Shou M, Lazarous DF, Scheinowitz M Biro S, Epstein SE and Unger EF: Angiogenic-induced enhancement of collateral blood flow to ischemic myocardium by vascular endothelial growth factor in dogs. Circulation 89 : 2183-2189, 1994.
20. Deuse T, Peter C, Fedak PW, Doyle T, Reichenspurner H, Zimmermann WH, Eschenhagen T, Stein W, Wu JC, Robbins RC, et al: Hepatocyte growth factor or vascular endothelial growth factor gene transfer maximizes mesenchymal stem cell-based myocardial salvage after acute myocardial infarction. Circulation 120 (Suppl 11): S247-S254, 2009.

21. Locatelli P, Olea FD, Hnatiuk A, De Lorenzi A, Cerdá M, Giménez CS, Sepúlveda D, Laguens R and Crottogini A: Mesenchymal stromal cells overexpressing vascular endothelial growth factor in ovine myocardial infarction. Gene Ther 22: 449-457, 2015.

22. Jiang S, Haider HK, Idris NM, Salim A and Ashraf M: Supportive interaction between cell survival signaling and angiocompetent factors enhances donor cell survival and promotes angiomyogenesis for cardiac repair. Circ Res 99: 776-784, 2006.

23. McMahon JM, Conroy S, Lyons M, Greiser U, O'shea C, Strappe P, Howard L, Murphy M, Barry F and O'Brien T: Gene transfer into rat mesenchymal stem cells: A comparative study of viral and nonviral vectors. Stem Cells Dev 15: 87-96, 2006.

24. Lindqvist LM and Vaux DL: BCL2 and related prosurvival proteins require $\mathrm{BAK} 1$ and $\mathrm{BAX}$ to affect autophagy. Autophagy 10: 1474-1475, 2014.

25. Boya P, González-Polo RA, Casares N, Perfettini JL, Dessen P, Larochette N, Métivier D, Meley D, Souquere S, Yoshimori T, et al: Inhibition of macroautophagy triggers apoptosis. Mol Cell Biol 25: 1025-1040, 2005.

26. Denton $\mathrm{D}, \mathrm{Xu} \mathrm{T}$ and Kumar S: Autophagy as a pro-death pathway. Immunol Cell Biol 93: 35-42, 2015.

27. Fitzwalter BE and Thorburn A: Recent insights into cell death and autophagy. FEBS J 282: 4279-4288, 2015.

28. Haider HK, Jiang S, Idris NM and Ashraf M: IGF-1-overexpressing mesenchymal stem cells accelerate bone marrow stem cell mobilization via paracrine activation of SDF-1alpha/CXCR4 signaling to promote myocardial repair. Circ Res 103: 1300-1308, 2008.

29. Fiedler J, Brill C, Blum WF and Brenner RE: IGF-I and IGF-II stimulate directed cell migration of bone-marrow-derived human mesenchymal progenitor cells. Biochem Biophys Res Commun 345: 1177-1183, 2006.

30. Song H, Song BW, Cha MJ, Choi IG and Hwang KC: Modification of mesenchymal stem cells for cardiac regeneration. Expert Opin Biol Ther 10: 309-319, 2010. 